\title{
Emergency Department Visits Between Inpatient Visits: An Overlooked Quality Measure?
}

\author{
Justin Craig Becker ${ }^{\mathrm{a}}$, Thomas Godfrey ${ }^{\mathrm{a}}$, Teresa Kern ${ }^{\mathrm{a}}$, Lindsey Ratliff ${ }^{\mathrm{b}}$, Nora Anderson ${ }^{\mathrm{c}}$
}

\begin{abstract}
Healthcare costs are rapidly accelerating in the United States and payers are looking for a solution to contain out-of-control expenditures. Readmissions can be easily measured thus making them an attractive metric of quality care. The Centers for Medicare and Medicaid Services (CMS) wants to minimize its financial outlay while pushing for increased quality. By this theory, if a patient remains out of the hospital, then the care delivered is considered to be high quality. Currently, emergency department (ED) visits between inpatient visits are not calculated into readmission rates. This review examined the sparse literature on this issue and proposed ideas to better examine readmissions as a quality measure overall. CMS has targeted patient readmissions to hospitals as a quality-based means of adjusting financial reimbursement. In 2013, the national patient readmission rate reduced from $19.5 \%$ to $17.5 \%$. This review determined that (a) the overlooked ED visits could be a metric for delivery of quality care; (b) decision analysis methods should be employed to determine whether or not these ED visits are actually a cost-effective means of keeping readmissions down and quality up; and (c) numerous process-based obstacles must be overcome before any reimbursement system would be impacted by ED visits. It is critical that changes to reimbursement or quality measures be fair and meaningful for providers and quality care for patients must remain the top priority to address ED visits between inpatient visits.
\end{abstract}

Keywords: Emergency Department, Patient Quality, Readmissions, Reimbursement

\section{Introduction}

Health care costs are rapidly accelerating in the United States and payers are looking for a solution to contain out-ofcontrol expenditures. The older, fee-for-service method rewards providers for the volume of their care, not the quality (James, 2012). The fee-for-service method is characterized by the providers encouraging the patient to enter the hospital to receive treatment through in-hospital coverage (van Gigch, 2013). Newer payment methods control volume of care by bundling payments or capitation. An additional method, payfor-performance, uses quality metrics as a means of calculating payment.

The Centers for Medicare and Medicaid Services (CMS) targeted patient readmissions to hospitals as a quality-based means of adjusting financial reimbursement. A readmission is any admission to the hospital within 30 days of initial inpatient discharge (CMS, 2014). Patient readmission metrics originated with the start of managed care. For years, more visits meant more payments to providers and hospitals.

CMS has sought to reduce readmissions to the hospital by threatening to reduce payments for the additional care (CMS, 2014). Not surprisingly where money is involved, they have succeeded. In 2013, the national patient readmission rate reduced from $19.5 \%$ to $17.5 \%$ (American Hospital Association, 2015). While certainly a welcomed improvement, it still leads one to question whether or not care quality has actually been improved, or have hospitals and providers found ways to get around the system. Inpatient hospital care is the most costly form of care. CMS wants to minimize its financial outlay while pushing for increased quality. By this theory, if a patient remains out of the hospital, then the care delivered is considered to be high quality.
Readmissions can be readily measured, thus making them an attractive metric of quality care. For example, an ideal method of monitoring admissions could be a computer program that tracks the discharge dates (California HealthCare Foundation, 2011). But is this an oversimplification? There are other costly treatment sources that do not require hospital admission. Since readmissions only count with regard to time between inpatient visits, there is a large missing factor in the quality equation --- visits to the emergency department (ED).

Currently, ED visits have no bearing on Medicare reimbursement rates for readmission. A patient can visit the $\mathrm{ED}$, be stabilized, and leave in the same day; the readmission process is avoided and thus the care is deemed "quality" care. By these standards, a patient admitted to the hospital 29 days later with no ED visits is considered to have received lower quality care than a patient with multiple ED visits but no formal readmission within 30 days.

Policymakers should rethink these standard measures and account for these ED visits. Receiving care in demanding emergency rooms is not without elevated risk to the patient. EDs are overloaded with patients that will be triaged on the perceived emergent nature of the problem. It is not the best place for thoughtful continuous care. Literature well documents issues related to patient release with abnormal vital signs, incomplete examination of the relevant organ, and/or incomplete chart history essential for treatment and diagnoses (The Sullivan Group, n.d.). Given the diverse conditions that incoming patients present with, mistakes can be made and more subtle considerations overlooked. Patients can be unintentionally exposed to a range of critical risk factors more often than in the conventional primary care setting.

a. Department of Public Health Sciences, Pennsylvania State University, College of Medicine

b. Department of Science Engineering \& Technology, Pennsylvania State University

c. Department of Natural Science \& Mathematics, Indiana University of Pennsylvania 
This paper will not seek to increase quality or mitigate costs in EDs. Rather, it will express how the current quality metric is deficient and propose ideas to better examine readmissions as a quality measure overall.

\section{Literature Review}

CMS has been judiciously looking at readmission rates (with regards to payment reduction) within the last few years. As a result, there is limited research on this topic. The literature reviewed identifies ED usage after specific procedures, or in a certain population with a chronic condition. These studies examined quality with regards to medical care. Generalized studies about ED usage after a hospital discharge as it pertained to policy and reimbursement were significantly limited. Three studies currently exist and therefore are noteworthy to be discussed.

Boston Medical Center. The study investigated the perspective of an individual urban hospital and analyzed concrete, internal data. Rising, White, Fernandez, and Boutwell (2013) conducted a retrospective cohort study from administrative data at the Boston Medical Center. The researchers analyzed data from the ED databases from January 1 to June 30, 2010. Inpatient discharges from January 1 to May 31, 2010 were examined and any May discharges were followed through the end of June to determine if a readmission had occurred within 30 days.

During the study, there were 15,519 living discharges, accounting for 11,976 unique patients. Almost a quarter of the discharges $(n=3,695,23.8 \%)$ led to an $E D$ visit within 30 days (Rising et al., 2013). On a patient level, out of the 11,976 unique patients, $21.3 \%(n=2,552)$ returned to the ED within 30 days. During the study timeframe, there were 4,077 ED visits that were associated with hospital discharges and almost half of these visits $(n=1,865,45.7 \%)$ led to an additional admission.

The researchers stated limitations that results lacked representativeness of the problem because the Boston Medical Center was an urban teaching hospital, generally caring for the sickest patients. Furthermore, this study could not account for visits to other hospitals so these data pertained to merely one institution. Despite these limitations, the study strongly suggested that ED visits after inpatient discharges were commonplace and needed to be examined with regards to quality and reimbursement.

California, Florida, and Nebraska. The study examined similar measures as the study at Boston Medical Center; however, it analyzed data across three states (California, Florida, and Nebraska) from the Healthcare Cost and Utilization Project (HCUP). These data were made available by the Agency for Healthcare Research and Quality (AHRQ). States were selected by the researchers because of the data quality and the data contained unique patient identifiers, allowing for effective longitudinal tracking.

The cohort contained 5,032,254 hospitalizations with $4,028,555$ unique patients. Within 30 days of discharge, $17.9 \%$ had at least one acute care encounter, $7.5 \%$ had at least one ED visit and $12.3 \%$ had at least one readmission (Vashi et al., 2013). While the numbers did differ with regards to the study at the Boston Medical Center, this study demonstrated that follow up care after an admission was a prominent and costly outcome.

National Medicare Database. The final study, conducted by Kocher, Nallamothu, Birkmeyer, and Dimick (2013) examined ED visits for Medicare patients within 30 days of discharge for six common inpatient surgical procedures. Unlike the previous two studies, this study sought to examine national level data to ensure applicable and generalizable results. The researchers reviewed data from the Outpatient and Carrier Standard Analytical Files and Medicare Provider Analysis and Review Files for 2005-2007. By using International Classification of Diseases Ninth Revision (ICD9) codes, the researchers extracted patients that received the following procedures: percutaneous coronary intervention, coronary artery bypass grafting, elective abdominal aortic aneurysm repair, back surgery, hip fracture repair, or colectomy. Inclusion criteria for the study identified patients as enrolled in Medicare Part A and Part B, a United States resident, and alive beyond the 30-day readmission timeframe.

After applying the determined criteria filters, 2,382,514 unique patients underwent $2,456,021$ procedures at 4,536 different hospitals (Kocher et al., 2013). Across all the identified procedures, $17.3 \%$ of patients had an ED visit within 30 days of being discharged. A sizeable number of patients $(n=105,953,4.4 \%)$ had multiple ED visits within 30 days of discharge and $14.4 \%$ of all patients $(n=343,826)$ were readmitted to the hospital.

Limitations to this study did exist. The researchers stated that the analysis was solely descriptive and not necessarily reflective of specific regional inadequacies. Meaning that other areas of the country had a more serious problem with ED visits after discharge while others were quite minimal. It was also noted that no means existed to determine whether ED visits were driven by patient's choice or by local practice patterns of providers. While each ED visit was recorded, perhaps each visit was not truly an indicator of poor care, but rather a lack of alternatives for post-operative care. Although the study's shortcomings were noteworthy, the data still reflected an overlooked problem for reconciling ED visits with regards to measuring quality care.

\section{Implications of an ED Visit}

While documented data on ED usage post inpatient discharge is fairly easy to obtain through patient records, the difficulty derives from determining the significance of the ED visit. Since EDs provide a range of services, one chief obstacle to evaluate ED visits between inpatient visits as a measure of quality is perhaps associating the visit with a specific discharge. Patients could come to the ED multiple times for different reasons. This is not a concrete indicator that each ED visit and/or possible readmission is a result of poor care from the previous discharge. Unfortunately, the present data collected does not discern between ED visits related or unrelated to previous inpatient discharges.

An additional hurdle in looking at this developing health care issue addresses whether or not the ED visit is warranted. Emergency Medical Treatment and Active Labor Act (EMTALA) Laws dictate that denial of patient evaluation and care is not an option. No one would argue the decision to help a patient if they were concerned about their current health state. Commonly, experienced emergency medicine workers realize patients regularly arrive to the $\mathrm{ED}$ with an issue that could be managed more effectively in the primary care setting. There is legal obligation to treat and stabilize individuals arriving to the ED. So, how can an ED visit after an inpatient discharge be effectively measured for quality if 
patients have unlimited access and can willingly go with minimal reason?

Finally, there is no way to definitively confirm if each readmission to a hospital or ED is an indication of poor quality care. Every person responds differently to treatment plans. Patients are not interchangeable parts. A possible scenario is that a patient could have an adverse reaction to care that would require a trip to the ED or even a readmission. While efforts should be taken to ensure that quality care is delivered, it would appear inappropriate to penalize a provider when a patient has a natural, unexpected biological response to a course of treatment. Until the system starts to account for this additional factor, ED visits within 30 days of inpatient discharges cannot be an accurate quality measure with reimbursement implications.

\section{Action Plan}

If pay-for-performance becomes the prominent payment model for healthcare, then payers must address the issues related to ED visits as a measure of quality with regards to Medicare reimbursement. There needs to be evidence-based methodology to determine if ED visits are related or not. Physicians must be trusted to determine if the visit and treatment provided to the patient was necessary, and if the visit was due to a natural adverse reaction or poor inpatient care. This could be as simple as a required documentation from the provider leading to a new billing code. As with any change, there will be opposition. Providers must have the capacity to quickly include, compartmentalize, and implement this information such as making additions to patient's electronic medical records (EMR) to support providers. By accounting for these issues, ED visits then become a valid measure of quality within 30 days of an inpatient discharge.

\section{Limitations}

There are limitations to measuring the number of ED visits within 30 days of an inpatient discharge. First, patients are not obligated to use only one ED or the ED of the hospital where they were discharged. Since patient care can be delivered at multiple locations spanning across multiple health systems, is there any realistic method to track patient discharge and their ED visit history? The answer lies within a universal EMR system that may be unachievable because of financial constraints. EMRs are expensive and there remain a number of hospitals and health systems that simply cannot afford the massive investment necessary for such a vast system overhaul (Lewis, 2012).

While finding means to treat patients more efficiently and cost-effectively, there remains concern that care could be downgraded in an effort to minimize readmissions to either the hospital or ED. Changed in care processes should be scrutinized. For example, will trends emerge where ED physicians find other means for providing care that does not involve admission to the ED or hospital? Will more observation rooms be added or perhaps full urgent care clinics pop up as hospitals look to spend less money treating patients and keep them out of the actual hospital?

Finally, when it comes to readmissions as a quality measure, could ED visits after inpatient discharges keep individuals out of the hospital through the current managed care system? ED care is expensive. However, it remains cheaper than inpatient care, possibly making ED visits within 30 days of inpatient discharge something welcomed by CMS. There is high importance on time sensitivity when deciding whether or not an ED patient will be discharged or admitted. Decision analysis methods should be employed to determine whether or not these ED visits were actually a cost-effective means of keeping readmissions down and quality up.

\section{Conclusion}

This review determined that the overlooked ED visits could be quality measures for delivery of quality care. Hospital readmissions are widely accepted as indicators of lower quality care that lead to more expense. ED visits between inpatient discharges are commonly neglected when reporting quality metrics. ED visits should be considered part of the metric when counting hospital readmissions. However, simply counting the number of ED visits within 30 days of inpatient discharge does not address the entire scope of this issue. This review exposes numerous process-based obstacles to be overcome before any reimbursement system would be impacted by ED visits. It is critical that changes to reimbursement or quality measures be fair and meaningful for providers. With the intent to treat patients, providers must do what is best for the patient without the fear of being marginalized as quality care remains the top priority.

\section{References}

American Hospital Association. (2015). Rethinking the hospital readmissions reduction

program. Trendwatch. Retrieved: 31 July 2015.

California HealthCare Foundation. (2011). Using tracking tools to improve patient flow in hospitals. Retrieved: 31 July 31, 2015. www.chcf.org

EHR implementation still cost too much (2012). Information: Connecting the healthcare technology community. Retrieved: 31 July 31, 2015 at

http://www.informationweek.com/healthcare/electronic-health records/ehr-still-costs-too-much/d/d-id/1105211

Kocher, K., Nallamothu, B., Birkmeyer, J., \& Dimick, J. (2013). Emergency department visits after surgery are common for Medicare patients, suggesting opportunities to improve care. Health Affairs. 32(9), 1600-1607.

Doi; 10.1377/hlthaff.2013.0067

Rising, K., White, L., Fernandez, W., Boutwell, A. (2013). Emergency department visits after hospital discharge: a missing part of the equation. Annals of Emergency Medicine. 62(2), 145-150. doi:10.1016/j.annemergmed.2013.01.024 The Sullivan Group (No Date). Ten reasons your emergency department may not be as safe as you think it is. Retrieved: 31 July 2015.

www.thesullivangroup.com/tsg_emri_10_reasons.pdf Van Gigch, J. P., (2013). Control and regulation. Systems Design Modeling and Metamodeling. Chapter 8, 207. Retrieved: 31, July 2015.

Vashi, A., Fox, J., Carr, B., D’Onofrio, G., Pines, J., Ross, J., \& Gross, C. (2013). Use of hospital-based acute care among patients recently discharged from the hospital. Journal of the American Medical Association. 309(4), 364-371. 